\title{
Nonlinear Diffusion Filtering for Classifying Image
}

\author{
A.Shehanaz ${ }^{1}$, A.Divya M.Tech ${ }^{2}$ \\ IFET College Of Engineering, Department Of Computer Science ,Villupuram, Tamilnadu, India ${ }^{1,2}$
}

\begin{abstract}
Image processing is a signal processing for which input is an image output of an image may be characteristics or parameter related to the image. Under this concept the image classification which has both foreground and background feature of an image with edges, lines flow lines structures in foreground and inhibits smooth clutter in background. In this paper the saliency driven nonlinear diffusion filtering algorithm is used and the image is classified using multiscale information fusion in original image, they are applied with diffusion process and finally mapped with saliency. Here the background image is considered as noise which improves image classification and finally they are removed using nonlinear diffusion filtering this process makes the classification of images in an more accurate formation. At larger scales the background is filtered out and the foreground is preserved .Various experimental test has been conducted for image classification using multiscale space such as PASCAL2005 and oxford17 flower dataset with high classification rates
\end{abstract}

Keywords: PASCAL2005, multiscale space, nonlinear diffusion filtering

\section{INTRODUCTION}

Image processing is interesting topic and they has image classification for classifying images, each image has an foreground image and a background image, where the foreground images are much clearer than the background but in image classification is difficult in classifying and they are treated as noise .In paper [1] zhang et al analysed that the background has some correlations with the foreground ,so both background and foreground yields less recognition when combined than using the foreground alone. Heintz and koller [2] showed the spatial context information used to detect objects. shotten et al[3] proposed algorithm used for recognizing and segmenting the object in images based on appearance, shape and the context of information,here the input image is segmented into regions and each were used to correct labels.In order to deal effectively with background,we use saliency driven nonlinear diffusion filtering to genetrate multiscale space, in which information at scales is highly complementary than the information at the other scales .A nonlinear diffusion[4] widely used in image denoising ,enhancement,etc. They can preserve or enhance important structures, such as edges and lines. We combine a saliency map, which marks saliency degrees of the individual pixels with nonlinear diffusion filtering,by saliency map to define weights of image gradients. Its clear that based on saliency map,the background regions corresponding to salient regions with important image structures .The saliency driven multiscale space of an image can be used to handle uncertain background information.

At larger scales ,background is filtered out and Foreground is preserved .At small scales ,background and foreground is preserved .If the background is context of foreground ,the images from same class may be similar at small scales than at larger scales. The weighted average is preferred to minimum of the distance at some representative scales.
The saliency driven nonlinear multiscale space image represent has adventages:

- The nonlinear space has important image structures which is preserved or it enhances at wider scales and the locations of important structures are not shifted at diffusion at any scale

- The background image regions which has fusion of information from different scales , the background scale is a context for the foreground or noise .

- The saliency driven nonlinear multiscale representations can be easily supplied as input to any existing image classification algorithms.

\section{SALIENCY DRIVEN NONLINEAR DIFFUSION}

They has linear and nonlinear diffusion filtering along the Gaussian scale space Space: Let $\mathrm{u}(\mathrm{x}, \mathrm{y}, \mathrm{t})$ be the grey value at position $(x, y)$ and scale $t$ in the multi-scale space. The image diffusion filtering is defined by the diffusion equation.

Nonlinear Diffusion: If the D in a function of evolving image The nonlinear diffusion preserves and enhances image structures defined by large gradient values.

If image structures with large gradients are all in the foreground, nonlinear diffusion filters out the background. However, there may be large image gradients in the background.

Thus, we propose a saliency driven nonlinear diffusion equation which blurs non-salient regions and preserves salient regions. 


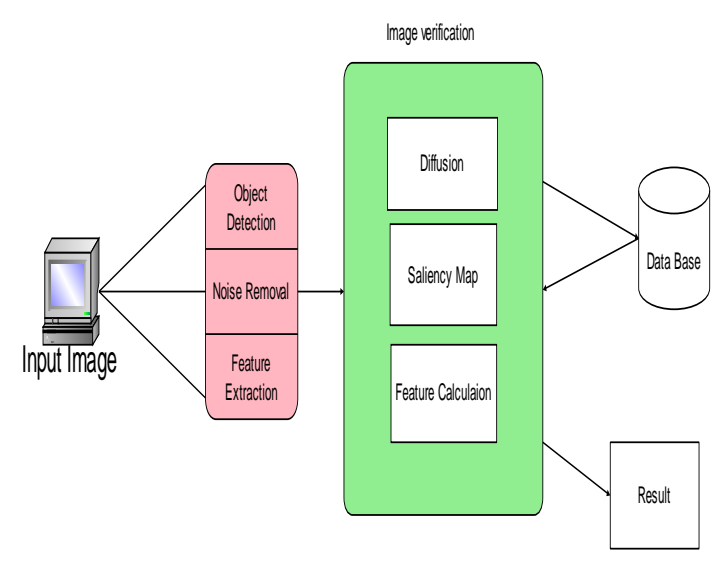

SALIENCY DETECTION

Saliency detection methods can be grouped as supervised and unsupervised, Here we first introduce the supervised methods and then the unsupervised methods. Finally, the method used in this paper is introduced. Supervised methods [5], [6] detect saliency using a classifier which is trained using samples for which saliency is well labeled. Marchesotti et al. [7] trained a classifier

for each target image using the images most similar to an annotated database to construct its saliency map. The underlying assumption is that images sharing a globally similar visual appearance are likely to share similar saliencies.This supervised saliency detection needs a very large well-labeled with an image database, which is not easy to obtain. Scale 0

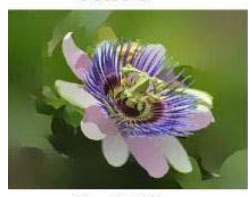

Scale 20

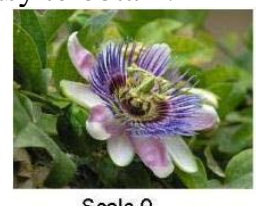

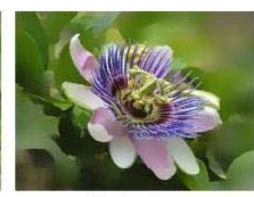
Scale 5

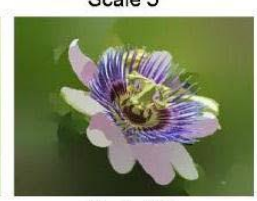

Scale 50

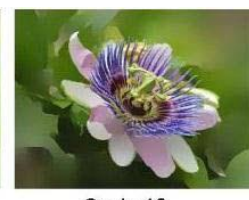
Scale 10

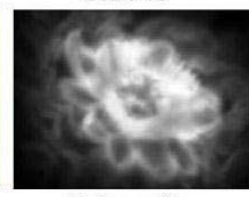

Unsupervised saliency detection [7], [8] usually starts with features of image structures known to be salient for the human visual system (HVS). These structure features include the intensity of salient regions, and the orientation, position.

- Local structures should be salient with respect to their surroundings [9].

- Frequently occurring features should be suppressed [10]

- $\quad$ The salient pixels should be grouped together, rather than scattered across the image.

\section{MULTISCALE IMAGE REPRESENT AND CLASSIFICATION}

To classify images using the saliency driven multi-scale image representation. The images used in foreground are highly clearer than background and more likely correctly classified at larger scales and the images in background are clearer more likely classified at small scale.So ,information from different scales can be fused to acquire more accurate image classification results

Our image classification framework which is shown as image is represented by its multi-scale images. Then, for each scale t, scale invariant feature transform features, which are widely used to represent image regions, are extracted, and the bag-of-words model is used to generate a word frequency histogram ht. The dissimilarity between images 1 and 2 at scale $t$ is represented by the .2 distance $\mathrm{d}(\mathrm{ht} 1, \mathrm{~h} 2 \mathrm{t})$ between histograms ht 1 and $\mathrm{h} 2 \mathrm{t}$.

\section{IMPLEMENTATION}

cale TM

-

cale T0

$\bullet$

cale $\mathrm{Tm}$

ultiscale diffusion

Scale TM:

This scale TM is the maximum scale at which the diffusion process converges and foreground and background is segmentation is completed.

\section{Scale T0:}

The inclusion of the original image corresponding to scale $\mathrm{T} 0$ in $\mathrm{T}(\mathrm{ie}) \mathrm{t}=\{\mathrm{TM}, \mathrm{T} 0, \mathrm{Tm}\}$ can provide a correction if the foreground is incorrectly filtered out and using the image at scale $\mathrm{Tm}$ alone is not sufficient to obtain a correct classification result.

\section{Scale Tm:}

The scaleTm is a midscale and it compromise between smoothing and background and preserves foreground

\section{MULTI SCALE DIFFUSION (SALIENCY)}

Saliency maps, the foreground regions were correctly detected. Our saliency driven nonlinear diffusion preserved their foreground regions and largely smoothed the background regions.

Therefore, at scales $T m$ and $T M$ in which the backgrounds were filtered out, the images were correctly classified. This produces a correct classification by multi-scale fusion.

The scales Tm, TM and multi-scale fusion are used but incorrectly classified at scale 0 .

Both the two original images contain large areas of background. In their saliency maps, the foreground regions were correctly detected.

Our saliency driven nonlinear diffusion preserved their foreground regions and largely smoothed the background regions. 


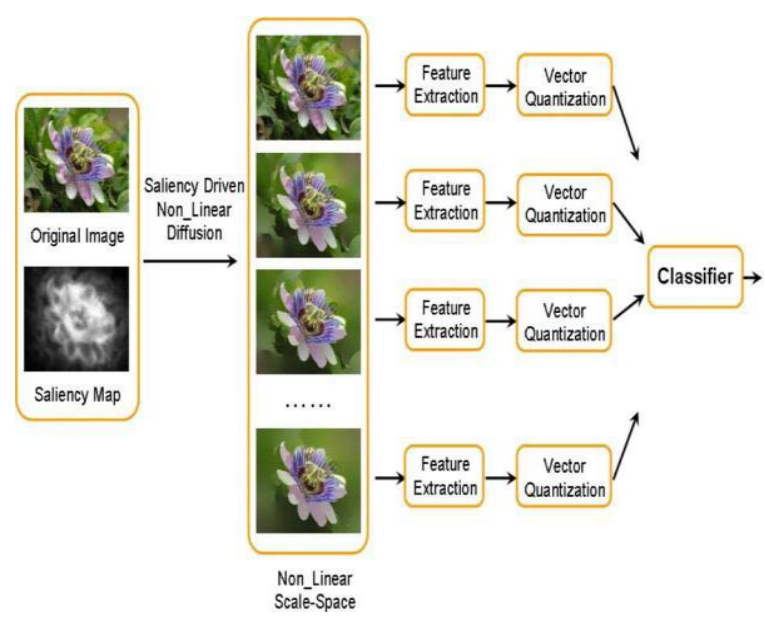

The saliency driven multi-scale fusion uses an background information .At large scale the background which is filtered out and the foreground which is preserved.At small scale ,both the background and the foreground regions are preserved. When the background is a context for foreground, then the images of same category are more similar at small scale.

When background which is noise ,the images are widely similar at large scale The distance between two images as a weighted average of the distances in the different used scales ,instead of the minimum distances at all scale the use of a weighted average reduces the classification in error also they has cases in which the foreground

We can extend our baseline by following:

- The saliency detection technique is combined with nonlinear diffusion filtering.

- Multi-scale fusion is used to combine the information

from the saliency driven nonlinear diffusion filtering.

- We apply the proposed filtering and fusion method to image classification. To our knowledge, there is no other work which applies nonlinear diffusion filtering to image classification.

\section{CONCLUSION}

The saliency driven multi-scale nonlinear diffusion filtering has been proposed by removing the background image which improves the classification of images. The saliency driven nonlinear multi-scale space preserves and even enhances important image local structures, such as lines and edges, at large scales. Multi-scale information has been fused using a weighted function of the distances between images at different scales. Experiments have been conducted on widely used datasets, namely the PASCAL2005 dataset, the Oxford 102 flowers dataset, and the Oxford 17 flowers dataset. The results have demonstrated that saliency driven multi-scale information fusion improves the accuracy of image classification.

\section{REFERENCES}

[1] J. Zhang, M. Marszalek, S. Lazebnik, and C. Schmid, "Local features and kernels for classification of texture and object categories: A comprehensive study," Int. J. Comput. Vis., vol. 73, no. 2, pp. 213-238, Jun. 2007.
[2] G. Heitz and D. Koller, "Learning spatial context: Using stuff to find things," in Proc. Eur. Conf. Comput. Vis., 2008, pp. 30-43.

[3] J. Shotton, J. Winn, C. Rother, and A. Criminisi, "Textonboost: Joint appearance, shape and context modeling for multi-class object recognition and segmentation," in Proc. Eur. Conf. Comput. Vis., 2006, pp. 1-15.

[4] J. Weickert, "A review of nonlinear diffusion filtering," in ScaleSpace Theory Compute Vision (Lecture Notes in Computer Science). New York, NY, USA: Springer-Verlag, 1997, pp. 1-28.

[5] T. Judd, K. A. Ehinger, F. Durand, and A. Torralba, "Learning to predict where humans look," in Proc. IEEE Int. Conf. Comput. Vis., Feb. 2009, pp. 2106-2113.

[6] H. Jiang, J. Wang, Z. Yuan, Y. Wu, N. Zheng, and S. Li, "Salient object detection: A discriminative regional feature integration approach," in Proc. IEEE Conf. Comput. Vis. Pattern Recognit., Jun. 2013, pp. 2083-2090.

[7] L. Marchesotti, C. Cifarelli, and G. Csurka, "A framework for visual saliency detection with applications to image thumb nailing," in Proc. IEEE 12th Int. Conf. Comput. Vis., Oct. 2009, pp. 2232-2239.

[8] J. Harel, C. Koch, and P. Perona, "Graph-based visual saliency," in Proc. Annu. Conf. Neural Inf. Process. Syst., 2007, pp. 545-552.

[9] X. Hou and L. Zhang, "Saliency detection: A spectral residual approach," in Proc. IEEE Conf. Comput. Vis. Pattern Recognit., Jun. 2007, pp. 1-8. 